\title{
SECOND ORDER MELNIKOV FUNCTIONS OF PIECEWISE HAMILTONIAN SYSTEMS
}

\author{
PEIXING YANG \\ School of Mathematical Sciences, Shanghai Jiaotong University, \\ Shanghai 200240, P. R. China \\ peixing0806@sjtu.edu.cn \\ JEAN-PIERRE FRANÇOISE * \\ Laboratoire Jacques-Louis Lions, Sorbonne Université, \\ UMR 7598 CNRS, 4 Place Jussieu, 75252, Paris Cedex, France \\ Jean-Pierre.Francoise@upmc.fr \\ JIANG YU \\ School of Mathematical Sciences, Shanghai Jiaotong University, \\ Shanghai 200240, P. R. China \\ jiangyu@sjtu.edu.cn
}

Received (to be inserted by publisher)

\begin{abstract}
In this paper, we consider the general perturbations of piecewise Hamiltonian systems. A formula for the second order Melnikov functions is derived when the first order Melnikov functions vanish. As an application, we can improve an upper bound of the number of bifurcated limit cycles of a piecewise Hamiltonian system with quadratic polynomial perturbations.
\end{abstract}

Keywords: Melnikov functions; Piecewise Hamiltonian system; Bifurcation; Limit cycles.

\section{Introduction and main results}

One of the most important topics in the classical problems for smooth system is the weak 16th Hilbert problem. In order to study limit cycles which are bifurcated from the perturbation of a center, more and more researchers study on piecewise systems. The study of piecewise differential systems goes back to Andronov and his coworkers[Andronov et al., 1966], and in the recent years, there are more and more papers studying on the piecewise linear differential systems. For readers, not yet acquainted with the subject, we refer to the book[Di Bernardo et al., 2008], and the survey[Makarenkov \& Lamb, 2012].

In 1990, Lum and Chua[Lum \& Chua, 1991] conjectured that in the case the plane is divided into two pieces by a straight line and the piecewise linear system is continuous and nonsmooth, there is at most one limit cycle. This was proved by E. Freire and his coworkers[Freire et al., 1998].

Recently, the discontinuous piecewise linear differential systems in the plane with two pieces separated by a straight line has been studied by many researchers. Han and Zhang[Han \& Zhang, 2010] conjectured that the maximum number of limit cycles in this case is two. But Huan and Yang [Huan \& Yang, 2013]

*Corresponding Author. 
gave a numerical evidence on the existence of three limit cycles. And Llibre and Ponce[Llibre \& Ponce, 2012] proved the existence of three limit cycles.

There are many techniques for computing analytically the periodic solutions of piecewise differential systems, such as first integrals, Poincaré map, averaging theory or Melnikov functions and so on.

The averaging theory of first order for piecewise differential systems is given by Llibre et al.[Llibre et al., 2015b]. Then Llibre and his coauthors[Llibre et al., 2015a] developed the averaging theory of first and second order for studying discontinuous piecewise systems in arbitrary dimension. The first order Melnikov function of piecewise Hamiltonian systems is derived by Liu and Han[Liu \& Han, 2010]. Cardin and Torregrosa[Cardin \& Torregrosa, 2016] studied the piecewise linear perturbations of a linear center, which is a generalization to piecewise linear systems of the extension to higher order perturbations and calculated its higher Melnikov functions up to sixth order. But to the best of our knowledge, there is no result about the higher order Melnikov function for the general perturbation of piecewise Hamiltonian systems.

So in the present paper, we derived a formula for the second order Melnikov function of the piecewise Hamiltonian systems in section 2 and 3. And we consider an example of perturbations for a linear center by piecewise quadratic polynomials in section 4 . Finally we make some remarks in section 5 .

\section{The second order Melnikov functions}

More precisely, we consider the perturbed system as follows,

$$
d H+\epsilon \omega=0
$$

where

$$
H(x, y)= \begin{cases}H^{+}(x, y), & x>0 \\ H^{-}(x, y), & x \leq 0\end{cases}
$$

and

$$
\omega= \begin{cases}\omega^{+}=P^{+}(x, y) d y-Q^{+}(x, y) d x, & x>0 \\ \omega^{-}=P^{-}(x, y) d y-Q^{-}(x, y) d x, & x \leq 0\end{cases}
$$

with $H^{ \pm}(x, y), P^{ \pm}(x, y), Q^{ \pm}(x, y) \in C^{\infty}$. When $\epsilon=0$, system (2.1) is called a piecewise Hamiltonian system. The $y$-axis $x=0$ is the discontinuity line.

Suppose that system (2.1) with $\epsilon=0$ has a family of periodic orbits near the origin. And we give the same assumption as [Liu \& Han, 2010]. Let $D$ be an interval, and there exist two points $A_{0}(h)=\left(0, a_{0}(h)\right)$ and $B_{0}(h)=\left(0, b_{0}(h)\right)$ on $x=0$, such that for $h \in D$,

$$
H^{+}\left(A_{0}(h)\right) \equiv H^{+}\left(B_{0}(h)\right) \equiv h, H^{-}\left(A_{0}(h)\right) \equiv H^{-}\left(B_{0}(h)\right),
$$

where $a_{0}(h) \neq b_{0}(h)$, which implies the unperturbed system has a family of periodic orbits $L_{h}=L_{h}^{+} \cup L_{h}^{-}$ where $L_{h}^{+}$is defined by $H^{+}(x, y)=h$ on $x>0$ beginning from $A_{0}(h)$ and ending at $B_{0}(h), L_{h}^{-}$is defined by $H^{-}(x, y)=H^{-}\left(B_{0}(h)\right)$ on $x \leq 0$ beginning from $B_{0}(h)$ and ending at $A_{0}(h)$. It is obvious that $L_{h}$ is piecewise smooth, see Figure 1.

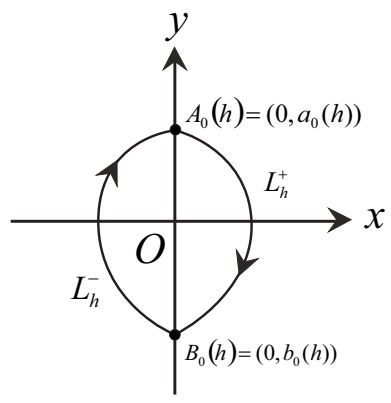

Fig. 1. Unperturbed system (2.1) with $\epsilon=0$. 
For the perturbed system (2.1), we consider a solution which starts from $A_{0}(h)$, and denote $B_{\epsilon}(h)=$ $\left(0, b_{\epsilon}(h)\right)$ be the first intersection point with the negative $y$-axis, and $A_{\epsilon}(h)=\left(0, a_{\epsilon}(h)\right)$ be the first intersection point with the positive $y$-axis, see Figure 2. Then we can define the bifurcation function as

$$
H^{+}\left(A_{\epsilon}\right)-H^{+}\left(A_{0}\right)=\epsilon M_{1}(h)+\epsilon^{2} M_{2}(h)+\cdots .
$$

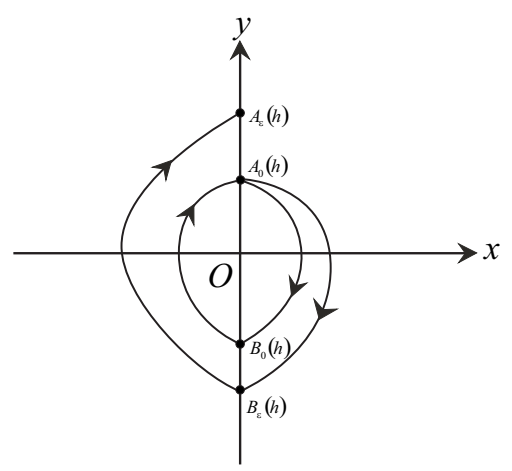

Fig. 2. Perturbed system (2.1).

As in the smooth case, we define $M_{1}(h)$ by the first order Melnikov function (cf Liu and Han [Liu \& Han, 2010]) and $M_{2}(h)$ by the second order Melnikov function. In the present paper, we give the expression of the second order Melnikov function for the piecewise Hamiltonian system.

The main theorem is the following.

Theorem 2.1. Consider system (2.1) with the assumption (2.2), and $M_{1}(h) \equiv 0$, the second order Melnikov function is given by

$$
\begin{aligned}
M_{2}(h) & =\frac{H_{y}^{+}\left(A_{0}\right)}{H_{y}^{-}\left(A_{0}\right)}\left[2 \left(\int_{\widehat{B_{0} A_{0}}} \psi^{-} \omega^{-}+\frac{H_{y}^{-}\left(B_{0}\right)}{H_{y}^{+}\left(B_{0}\right)} \psi^{-}\left(B_{0}\right) \int_{\widehat{A_{0} B_{0}}} \omega^{+}\right.\right. \\
& \left.\left.-\frac{P^{-}\left(B_{0}\right)}{H_{y}^{+}\left(B_{0}\right)} \int_{\widehat{A_{0} B_{0}}} \omega^{+}\right)+\frac{H_{y y}^{-}\left(B_{0}\right)}{H_{y}^{-2}\left(B_{0}\right)}\left(\int_{\widehat{B_{0} A_{0}}} \omega^{-}\right)^{2}\right] \\
& +\frac{H_{y}^{+}\left(A_{0}\right)}{H_{y}^{-}\left(A_{0}\right)} \frac{H_{y}^{-}\left(B_{0}\right)}{H_{y}^{+}\left(B_{0}\right)}\left[2 \left(\int_{\widehat{A_{0} B_{0}}} \psi^{+} \omega^{+}+\frac{P^{+}\left(B_{0}\right)}{H_{y}^{+}\left(B_{0}\right)} \int_{\widehat{A_{0} B_{0}}} \omega^{+}\right.\right. \\
& \left.\left.-\psi^{+}\left(B_{0}\right) \int_{\widehat{A_{0} B_{0}}} \omega^{+}\right)-\frac{H_{y y}^{+}\left(B_{0}\right)}{H_{y}^{+2}\left(B_{0}\right)}\left(\int_{\widehat{A_{0} B_{0}}} \omega^{+}\right)^{2}\right],
\end{aligned}
$$

with $\psi^{ \pm}$are defined by

$$
\psi^{ \pm}(x, y)=\int_{0}^{T^{ \pm}(x, y)} \operatorname{div}\left(\chi^{ \pm}\right) \circ\left(\varphi_{t}^{ \pm}\left(x_{0}^{ \pm}, y_{0}^{ \pm}\right)\right) d t,
$$

where $\chi^{ \pm}=\left(P^{ \pm}, Q^{ \pm}\right)$and $\varphi_{t}^{ \pm}$are the solution of system (2.1) passing through $\left(x_{0}^{ \pm}, y_{0}^{ \pm}\right)$at $t=0$ and end at $(x, y)$ with $t=T^{ \pm}(x, y)$.

If we consider the system with the perturbations up to second order in $\epsilon$, that is,

$$
\begin{cases}d H^{+}(x, y)+\epsilon \omega_{0}^{+}+\epsilon^{2} \omega_{1}^{+}=0, & x>0 \\ d H^{-}(x, y)+\epsilon \omega_{0}^{-}+\epsilon^{2} \omega_{1}^{-}=0, & x \leq 0\end{cases}
$$

where

$$
\omega_{0}^{ \pm}=P_{0}^{ \pm}(x, y) d y-Q_{0}^{ \pm}(x, y) d x, \quad \omega_{1}^{ \pm}=P_{1}^{ \pm}(x, y) d y-Q_{1}^{ \pm}(x, y) d x .
$$

Then we have the following corollary: 
Corollary 2.1. System (2.5) with the assumption (2.2), and $M_{1}(h) \equiv 0$, the second order Melnikov function is given by

$$
\begin{aligned}
M_{2}(h) & =\frac{H_{y}^{+}\left(A_{0}\right)}{H_{y}^{-}\left(A_{0}\right)}\left[2 \left(\int_{\widehat{B_{0} A_{0}}} \psi^{-} \omega_{0}^{-}-\omega_{1}^{-}+\frac{H_{y}^{-}\left(B_{0}\right)}{H_{y}^{+}\left(B_{0}\right)} \psi^{-}\left(B_{0}\right) \int_{\widehat{A_{0} B_{0}}} \omega_{0}^{+}\right.\right. \\
& \left.\left.-\frac{P_{0}^{-}\left(B_{0}\right)}{H_{y}^{+}\left(B_{0}\right)} \int_{\widehat{A_{0} B_{0}}} \omega_{0}^{+}\right)+\frac{H_{y y}^{-}\left(B_{0}\right)}{H_{y}^{-2}\left(B_{0}\right)}\left(\int_{\widehat{B_{0} A_{0}}} \omega_{0}^{-}\right)^{2}\right] \\
& +\frac{H_{y}^{+}\left(A_{0}\right)}{H_{y}^{-}\left(A_{0}\right)} \frac{H_{y}^{-}\left(B_{0}\right)}{H_{y}^{+}\left(B_{0}\right)}\left[2 \left(\int_{\widehat{A_{0} B_{0}}} \psi^{+} \omega_{0}^{+}-\omega_{1}^{+}+\frac{P_{0}^{+}\left(B_{0}\right)}{H_{y}^{+}\left(B_{0}\right)} \int_{\widehat{A_{0} B_{0}}} \omega_{0}^{+}\right.\right. \\
& \left.\left.-\psi^{+}\left(B_{0}\right) \int_{\widehat{A_{0} B_{0}}} \omega_{0}^{+}\right)-\frac{H_{y y}^{+}\left(B_{0}\right)}{H_{y}^{+2}\left(B_{0}\right)}\left(\int_{\widehat{A_{0} B_{0}}} \omega_{0}^{+}\right)^{2}\right],
\end{aligned}
$$

where $\psi^{ \pm}, \chi^{ \pm}, \varphi_{t}^{ \pm}$and $T^{ \pm}(x, y)$ are defined as the same with Theorem 2.1.

\section{The proof of main Theorem}

In this section, we give the proof of the main theorem.

Proof. Split the formula (2.3) into four parts as follows

$$
\begin{aligned}
H^{+}\left(A_{\epsilon}\right)-H^{+}\left(A_{0}\right) & =\left[H^{+}\left(A_{\epsilon}\right)-H^{-}\left(A_{\epsilon}\right)\right]+\left[H^{-}\left(A_{\epsilon}\right)-H^{-}\left(B_{\epsilon}\right)\right] \\
& +\left[H^{-}\left(B_{\epsilon}\right)-H^{+}\left(B_{\epsilon}\right)\right]+\left[H^{+}\left(B_{\epsilon}\right)-H^{+}\left(A_{0}\right)\right] \\
& \equiv l_{1}+l_{2}+l_{3}+l_{4} .
\end{aligned}
$$

It is easy to calculate that

$$
\begin{aligned}
l_{4} & =H^{+}\left(B_{0}\right)-H^{+}\left(A_{0}\right)+\left.\epsilon H_{y}^{+}\left(B_{0}\right) \frac{\partial b_{\epsilon}}{\partial \epsilon}\right|_{\epsilon=0}+\left.\frac{1}{2} \epsilon^{2} H_{y}^{+}\left(B_{0}\right) \frac{\partial^{2} b_{\epsilon}}{\partial \epsilon^{2}}\right|_{\epsilon=0} \\
& +\frac{1}{2} H_{y y}^{+}\left(B_{0}\right) \epsilon^{2}\left(\left.\frac{\partial b_{\epsilon}}{\partial \epsilon}\right|_{\epsilon=0}\right)^{2}+O\left(\epsilon^{3}\right) .
\end{aligned}
$$

Then by the above formula, we have

$$
\begin{aligned}
\left.\frac{\partial l_{4}}{\partial \epsilon}\right|_{\epsilon=0} & =\left.H_{y}^{+}\left(B_{0}\right) \frac{\partial b_{\epsilon}}{\partial \epsilon}\right|_{\epsilon=0}, \\
\left.\frac{\partial^{2} l_{4}}{\partial \epsilon^{2}}\right|_{\epsilon=0} & =\left.H_{y}^{+}\left(B_{0}\right) \frac{\partial^{2} b_{\epsilon}}{\partial \epsilon^{2}}\right|_{\epsilon=0}+H_{y y}^{+}\left(B_{0}\right)\left(\left.\frac{\partial b_{\epsilon}}{\partial \epsilon}\right|_{\epsilon=0}\right)^{2} .
\end{aligned}
$$

Similarly, by $l_{2}=H^{-}\left(A_{\epsilon}\right)-H^{-}\left(B_{\epsilon}\right), \quad A_{\epsilon}=\left(0, a_{\epsilon}\right)$ and $B_{\epsilon}=\left(0, b_{\epsilon}\right)$, we can obtain

$$
\begin{aligned}
l_{2} & =H^{-}\left(A_{\epsilon}\right)-H^{-}\left(B_{\epsilon}\right) \\
& =H^{-}\left(A_{0}\right)+H_{y}^{-}\left(A_{0}\right)\left(\left.\epsilon \frac{\partial a_{\epsilon}}{\partial \epsilon}\right|_{\epsilon=0}+\left.\frac{1}{2} \epsilon^{2} \frac{\partial^{2} a_{\epsilon}}{\partial \epsilon^{2}}\right|_{\epsilon=0}+O\left(\epsilon^{3}\right)\right) \\
& +\frac{1}{2} H_{y y}^{-}\left(A_{0}\right)\left(\left.\epsilon \frac{\partial a_{\epsilon}}{\partial \epsilon}\right|_{\epsilon=0}+O\left(\epsilon^{2}\right)\right)^{2} \\
& -H^{-}\left(B_{0}\right)-H_{y}^{-}\left(B_{0}\right)\left(\left.\epsilon \frac{\partial b_{\epsilon}}{\partial \epsilon}\right|_{\epsilon=0}+\left.\frac{1}{2} \epsilon^{2} \frac{\partial^{2} b_{\epsilon}}{\partial \epsilon^{2}}\right|_{\epsilon=0}+O\left(\epsilon^{3}\right)\right) \\
& -\frac{1}{2} H_{y y}^{-}\left(B_{0}\right)\left(\left.\epsilon \frac{\partial b_{\epsilon}}{\partial \epsilon}\right|_{\epsilon=0}+O\left(\epsilon^{2}\right)\right)^{2}
\end{aligned}
$$

The formula (3.3) gives to

$$
\begin{aligned}
\left.\frac{\partial l_{2}}{\partial \epsilon}\right|_{\epsilon=0} & =\left.H_{y}^{-}\left(A_{0}\right) \frac{\partial a_{\epsilon}}{\partial \epsilon}\right|_{\epsilon=0}-\left.H_{y}^{-}\left(B_{0}\right) \frac{\partial b_{\epsilon}}{\partial \epsilon}\right|_{\epsilon=0}, \\
\left.\frac{\partial^{2} l_{2}}{\partial \epsilon^{2}}\right|_{\epsilon=0} & =\left.H_{y}^{-}\left(A_{0}\right) \frac{\partial^{2} a_{\epsilon}}{\partial \epsilon^{2}}\right|_{\epsilon=0}+H_{y y}^{-}\left(A_{0}\right)\left(\left.\frac{\partial a_{\epsilon}}{\partial \epsilon}\right|_{\epsilon=0}\right)^{2} \\
& -\left.H_{y}^{-}\left(B_{0}\right) \frac{\partial^{2} b_{\epsilon}}{\partial \epsilon^{2}}\right|_{\epsilon=0}-H_{y y}^{-}\left(B_{0}\right)\left(\left.\frac{\partial b_{\epsilon}}{\partial \epsilon}\right|_{\epsilon=0}\right)^{2} .
\end{aligned}
$$


As the same calculation, we can give the formulas as follows,

$$
\begin{aligned}
\left.\frac{\partial l_{1}}{\partial \epsilon}\right|_{\epsilon=0} & =\left.H_{y}^{+}\left(A_{0}\right) \frac{\partial a_{\epsilon}}{\partial \epsilon}\right|_{\epsilon=0}-\left.H_{y}^{-}\left(A_{0}\right) \frac{\partial a_{\epsilon}}{\partial \epsilon}\right|_{\epsilon=0}, \\
\left.\frac{\partial l_{3}}{\partial \epsilon}\right|_{\epsilon=0} & =\left.H_{y}^{-}\left(B_{0}\right) \frac{\partial b_{\epsilon}}{\partial \epsilon}\right|_{\epsilon=0}-\left.H_{y}^{+}\left(B_{0}\right) \frac{\partial b_{\epsilon}}{\partial \epsilon}\right|_{\epsilon=0}, \\
\left.\frac{\partial^{2} l_{1}}{\partial \epsilon^{2}}\right|_{\epsilon=0} & =\left.H_{y}^{+}\left(A_{0}\right) \frac{\partial^{2} a_{\epsilon}}{\partial \epsilon^{2}}\right|_{\epsilon=0}+H_{y y}^{+}\left(A_{0}\right)\left(\left.\frac{\partial a_{\epsilon}}{\partial \epsilon}\right|_{\epsilon=0}\right)^{2} \\
& -\left.H_{y}^{-}\left(A_{0}\right) \frac{\partial^{2} a_{\epsilon}}{\partial \epsilon^{2}}\right|_{\epsilon=0}-H_{y y}^{-}\left(A_{0}\right)\left(\left.\frac{\partial a_{\epsilon}}{\partial \epsilon}\right|_{\epsilon=0}\right)^{2}, \\
\left.\frac{\partial^{2} l_{3}}{\partial \epsilon^{2}}\right|_{\epsilon=0} & =\left.H_{y}^{-}\left(B_{0}\right) \frac{\partial^{2} b_{\epsilon}}{\partial \epsilon^{2}}\right|_{\epsilon=0}+H_{y y}^{-}\left(B_{0}\right)\left(\left.\frac{\partial b_{\epsilon}}{\partial \epsilon}\right|_{\epsilon=0}\right)^{2} \\
& -\left.H_{y}^{+}\left(B_{0}\right) \frac{\partial^{2} b_{\epsilon}}{\partial \epsilon^{2}}\right|_{\epsilon=0}-H_{y y}^{+}\left(B_{0}\right)\left(\left.\frac{\partial b_{\epsilon}}{\partial \epsilon}\right|_{\epsilon=0}\right)^{2} .
\end{aligned}
$$

By (3.2), we have

$$
\begin{aligned}
\left.\frac{\partial b_{\epsilon}}{\partial \epsilon}\right|_{\epsilon=0} & =\left.\frac{1}{H_{y}^{+}\left(B_{0}\right)} \frac{\partial l_{4}}{\partial \epsilon}\right|_{\epsilon=0}, \\
\left.\frac{\partial^{2} b_{\epsilon}}{\partial \epsilon^{2}}\right|_{\epsilon=0} & =\frac{1}{H_{y}^{+}\left(B_{0}\right)}\left[\left.\frac{\partial^{2} l_{4}}{\partial \epsilon^{2}}\right|_{\epsilon=0}-H_{y y}^{+}\left(B_{0}\right)\left(\left.\frac{\partial b_{\epsilon}}{\partial \epsilon}\right|_{\epsilon=0}\right)^{2}\right] .
\end{aligned}
$$

As the same, (3.6) and (3.4) give rise to

$$
\begin{aligned}
\left.\frac{\partial a_{\epsilon}}{\partial \epsilon}\right|_{\epsilon=0} & =\frac{1}{H_{y}^{-}\left(A_{0}\right)}\left[\left.\frac{\partial l_{2}}{\partial \epsilon}\right|_{\epsilon=0}+\left.\frac{H_{y}^{-}\left(B_{0}\right)}{H_{y}^{+}\left(B_{0}\right)} \frac{\partial l_{4}}{\partial \epsilon}\right|_{\epsilon=0}\right], \\
\left.\frac{\partial^{2} a_{\epsilon}}{\partial \epsilon^{2}}\right|_{\epsilon=0} & =\frac{1}{H_{y}^{-}\left(A_{0}\right)}\left[\left.\frac{\partial^{2} l_{2}}{\partial \epsilon^{2}}\right|_{\epsilon=0}+\left.H_{y}^{-}\left(B_{0}\right) \frac{\partial^{2} b_{\epsilon}}{\partial \epsilon^{2}}\right|_{\epsilon=0}\right. \\
& \left.+H_{y y}^{-}\left(B_{0}\right)\left(\left.\frac{\partial b_{\epsilon}}{\partial \epsilon}\right|_{\epsilon=0}\right)^{2}-H_{y y}^{-}\left(A_{0}\right)\left(\left.\frac{\partial a_{\epsilon}}{\partial \epsilon}\right|_{\epsilon=0}\right)^{2}\right] .
\end{aligned}
$$

On the other hand, it is obvious that

$$
l_{4}=H^{+}\left(B_{\epsilon}\right)-H^{+}\left(A_{0}\right)=\int_{\widehat{A_{0} B_{\epsilon}}} d H^{+}=-\epsilon \int_{\widehat{A_{0} B_{0}}} \omega^{+}+O\left(\epsilon^{2}\right) .
$$

Then we have

$$
\left.\frac{\partial l_{4}}{\partial \epsilon}\right|_{\epsilon=0}=-\int_{\widehat{A_{0} B_{0}}} \omega^{+}
$$

With the same calculation,

$$
l_{2}=-\epsilon \int_{\widehat{B_{0} A_{0}}} \omega^{-}+O\left(\epsilon^{2}\right)
$$

which implies

$$
\left.\frac{\partial l_{2}}{\partial \epsilon}\right|_{\epsilon=0}=-\int_{\widehat{B_{0} A_{0}}} \omega^{-} .
$$

Hence by (2.3), (3.1) and (3.5), we have

$$
\begin{aligned}
M_{1}(h) & =\left.\sum_{i=1}^{4} \frac{\partial l_{i}}{\partial \epsilon}\right|_{\epsilon=0} \\
& =\left.\frac{\partial l_{2}}{\partial \epsilon}\right|_{\epsilon=0}+\left.\frac{\partial l_{4}}{\partial \epsilon}\right|_{\epsilon=0}+\left.H_{y}^{-}\left(B_{0}\right) \frac{\partial b_{\epsilon}}{\partial \epsilon}\right|_{\epsilon=0}-\left.H_{y}^{+}\left(B_{0}\right) \frac{\partial b_{\epsilon}}{\partial \epsilon}\right|_{\epsilon=0} \\
& +\left.H_{y}^{+}\left(A_{0}\right) \frac{\partial a_{\epsilon}}{\partial \epsilon}\right|_{\epsilon=0}-\left.H_{y}^{-}\left(A_{0}\right) \frac{\partial a_{\epsilon}}{\partial \epsilon}\right|_{\epsilon=0} .
\end{aligned}
$$


Substituting (3.6)-(3.9) into (3.10) leads to

$$
M_{1}(h)=-\frac{H_{y}^{+}\left(A_{0}\right)}{H_{y}^{-}\left(A_{0}\right)}\left[\frac{H_{y}^{-}\left(B_{0}\right)}{H_{y}^{+}\left(B_{0}\right)} \int_{\widehat{A_{0} B_{0}}} \omega^{+}+\int_{\widehat{B_{0} A_{0}}} \omega^{-}\right] .
$$

The formula (3.11) is given by Liu and Han[Liu \& Han, 2010], and if $M_{1}(h) \equiv 0$, from (3.11) we can find $\left.\frac{\partial l_{2}}{\partial \epsilon}\right|_{\epsilon=0}+\left.\frac{H_{y}^{-}\left(B_{0}\right)}{H_{y}^{+}\left(B_{0}\right)} \frac{\partial l_{4}}{\partial \epsilon}\right|_{\epsilon=0} \equiv 0$, namely,

$$
\left.\frac{\partial a_{\epsilon}}{\partial \epsilon}\right|_{\epsilon=0} \equiv 0
$$

Denote $\varphi_{t}^{+}=\varphi^{+}(t, x, y)$ be a solution of system $\left.(2.1)\right|_{\epsilon=0}$ on the half plane of $x>0$ starting from $\left(x_{0}^{+}, y_{0}^{+}\right)=A_{0}\left(0, a_{0}(h)\right)$ and define a function $\psi^{+}(x, y)$ as follows

$$
\psi^{+}(x, y)=\int_{0}^{T^{+}(x, y)} \operatorname{div}\left(\chi^{+}\right) \circ\left(\varphi_{t}^{+}\left(x_{0}^{+}, y_{0}^{+}\right)\right) d t
$$

where $\chi^{+}=\left(P^{+}(x, y), Q^{+}(x, y)\right)$ and $(x, y)=\varphi^{+}\left(T^{+}(x, y), x_{0}^{+}, y_{0}^{+}\right)$.

Denote $\chi_{0}^{+}$be the vector field of system (2.1) with $\epsilon=0$ on $x>0$, namely, $\chi_{0}^{+}=H_{y}^{+} \frac{\partial}{\partial x}-H_{x}^{+} \frac{\partial}{\partial y}$. In other words, $\psi^{+}$is the integral of the function $\operatorname{div}\left(\chi^{+}\right)$along the trajectories of $\chi_{0}^{+}$. Hence, the derivative of $\psi^{+}$along the trajectories of the vector field $\chi_{0}^{+}$denoted as $\chi_{0}^{+} \cdot\left(\psi^{+}\right)$satisfies $\chi_{0}^{+} \cdot\left(\psi^{+}\right)=\operatorname{div}\left(\chi^{+}\right)$. This yields,

$$
d H^{+} \wedge d \psi^{+}=\operatorname{div}\left(\chi^{+}\right) d x \wedge d y .
$$

On the other hand, $\operatorname{div}\left(\chi^{+}\right) d x \wedge d y=-d \omega^{+}$. It is obvious that

$$
\epsilon d H^{+} \wedge d \psi^{+}=\epsilon\left(-d\left(\psi^{+} d H^{+}\right)\right)=-\epsilon d \omega^{+} .
$$

Hence the 1-form $\omega^{+}-\psi^{+} d H^{+}$is closed. The domain on which it is defined being simply connected, there exists a unique function of $(x, y), R^{+}$(up to a constant that we fix with the condition $\left.R^{+}\right|_{x=0}=0$ ) so that:

$$
\omega^{+}=\psi^{+} d H^{+}+d R^{+}
$$

Next we consider the following equality, as in the smooth case (cf. [Françoise, 1996]),

$$
\left(1-\epsilon \psi^{+}\right)\left(d H^{+}+\epsilon \omega^{+}\right)=0,
$$

by integrating (3.13) over $\widehat{A_{0} B_{\epsilon}}$, which is

$$
\int_{\widehat{A_{0} B_{\epsilon}}} d\left(H^{+}+\epsilon R^{+}\right)=\epsilon^{2} \int_{\widehat{A_{0} B_{\epsilon}}} \psi^{+} \omega^{+} .
$$

After a simple calculation, we have

$$
H^{+}\left(B_{\epsilon}\right)-H^{+}\left(A_{0}\right)+\epsilon R^{+}\left(B_{\epsilon}\right)-\epsilon R^{+}\left(A_{0}\right)=\epsilon^{2} \int_{\widehat{A_{0} B_{0}}} \psi^{+} \omega^{+}+O\left(\epsilon^{3}\right) .
$$

According to (3.1), it is easy to obtain

$$
l_{4}+\epsilon\left[R^{+}\left(B_{0}\right)+\left.R_{y}^{+}\left(B_{0}\right) \frac{\partial b_{\epsilon}}{\partial \epsilon}\right|_{\epsilon=0} \epsilon+O\left(\epsilon^{2}\right)-R^{+}\left(A_{0}\right)\right]=\epsilon^{2} \int_{\widehat{A_{0} B_{0}}} \psi^{+} \omega^{+}+O\left(\epsilon^{3}\right) .
$$

From the above formula, we have

$$
\left.\frac{\partial^{2} l_{4}}{\partial \epsilon^{2}}\right|_{\epsilon=0}=-\left.2 R_{y}^{+}\left(B_{0}\right) \frac{\partial b_{\epsilon}}{\partial \epsilon}\right|_{\epsilon=0}+2 \int_{\widehat{A_{0} B_{0}}} \psi^{+} \omega^{+} .
$$

Combining (3.2) and (3.14) leads to

$$
\left.\frac{\partial^{2} b_{\epsilon}}{\partial \epsilon^{2}}\right|_{\epsilon=0}=\frac{1}{H_{y}^{+}\left(B_{0}\right)}\left[2\left(\int_{\widehat{A_{0} B_{0}}} \psi^{+} \omega^{+}-\left.R_{y}^{+}\left(B_{0}\right) \frac{\partial b_{\epsilon}}{\partial \epsilon}\right|_{\epsilon=0}\right)-H_{y y}^{+}\left(B_{0}\right)\left(\left.\frac{\partial b_{\epsilon}}{\partial \epsilon}\right|_{\epsilon=0}\right)^{2}\right] .
$$


Similarly, denote $\varphi_{t}^{-}=\varphi^{-}(t, x, y)$ be a solution of system $\left.(2.1)\right|_{\epsilon=0}$ on $x \leq 0$ starting from $\left(x_{0}^{-}, y_{0}^{-}\right)=$ $B_{\epsilon}\left(0, b_{\epsilon}(h)\right)$ and define a function $\psi^{-}(x, y)$ as follows

$$
\psi^{-}(x, y)=\int_{0}^{T^{-}(x, y)} \operatorname{div}\left(\chi^{-}\right) \circ\left(\varphi_{t}^{-}\left(x_{0}^{-}, y_{0}^{-}\right)\right) d t,
$$

where $\chi^{-}=\left(P^{-}, Q^{-}\right)$and $T^{-}(x, y)$ is the time of the solution ending at $(x, y)$.

Denote $\chi_{0}^{-}$be the vector field of system $(2.1)$ with $\epsilon=0$ on $x \leq 0$, namely, $\chi_{0}^{-}=H_{y}^{-} \frac{\partial}{\partial x}-H_{x}^{-} \frac{\partial}{\partial y}$, then we have $\chi_{0}^{-} \cdot\left(\psi^{-}\right)=\operatorname{div}\left(\chi^{-}\right)$, which implies

$$
d H^{-} \wedge d \psi^{-}=\operatorname{div}\left(\chi^{-}\right) d x \wedge d y .
$$

On the other hand, $\operatorname{div}\left(\chi^{-}\right) d x \wedge d y=-d \omega^{-}$, from which we can get

$$
\omega^{-}=\psi^{-} d H^{-}+d R^{-}
$$

where $R^{-}$is a function of $(x, y)$.

Similarly integrating the following equation over $\widehat{B_{\epsilon} A_{\epsilon}}$,

$$
\left(1-\epsilon \psi^{-}\right)\left(d H^{-}+\epsilon \omega^{-}\right)=0
$$

we have

$$
H^{-}\left(A_{\epsilon}\right)-H^{-}\left(B_{\epsilon}\right)+\epsilon R^{-}\left(A_{\epsilon}\right)-\epsilon R^{-}\left(B_{\epsilon}\right)=\epsilon^{2} \int_{\widehat{B_{0} A_{0}}} \psi^{-} \omega^{-}+O\left(\epsilon^{3}\right)
$$

which leads to

$$
\begin{aligned}
& l_{2}+\epsilon\left[R^{-}\left(A_{0}\right)+\left.R_{y}^{-}\left(A_{0}\right) \frac{\partial a_{\epsilon}}{\partial \epsilon}\right|_{\epsilon=0} \epsilon-R^{-}\left(B_{0}\right)-\left.R_{y}^{-}\left(B_{0}\right) \frac{\partial b_{\epsilon}}{\partial \epsilon}\right|_{\epsilon=0} \epsilon+O\left(\epsilon^{2}\right)\right] \\
= & \epsilon^{2} \int_{\widehat{B_{0} A_{0}}} \psi^{-} \omega^{-}+O\left(\epsilon^{3}\right) .
\end{aligned}
$$

From the above formula, and the condition that $M_{1}(h) \equiv 0$, we have

$$
\begin{aligned}
\left.\frac{\partial^{2} l_{2}}{\partial \epsilon^{2}}\right|_{\epsilon=0} & =-\left.2 R_{y}^{-}\left(A_{0}\right) \frac{\partial a_{\epsilon}}{\partial \epsilon}\right|_{\epsilon=0}+\left.2 R_{y}^{-}\left(B_{0}\right) \frac{\partial b_{\epsilon}}{\partial \epsilon}\right|_{\epsilon=0}+2 \int_{\widehat{B_{0} A_{0}}} \psi^{-} \omega^{-} \\
& =\left.2 R_{y}^{-}\left(B_{0}\right) \frac{\partial b_{\epsilon}}{\partial \epsilon}\right|_{\epsilon=0}+2 \int_{\widehat{B_{0} A_{0}}} \psi^{-} \omega^{-} .
\end{aligned}
$$

From (3.4) and (3.16), we have

$$
\begin{aligned}
\left.\frac{\partial^{2} a_{\epsilon}}{\partial \epsilon^{2}}\right|_{\epsilon=0} & =\frac{1}{H_{y}^{-}\left(A_{0}\right)}\left[2\left(\int_{\widehat{B_{0} A_{0}}} \psi^{-} \omega^{-}+\left.R_{y}^{-}\left(B_{0}\right) \frac{\partial b_{\epsilon}}{\partial \epsilon}\right|_{\epsilon=0}\right)+\left.H_{y}^{-}\left(B_{0}\right) \frac{\partial^{2} b_{\epsilon}}{\partial \epsilon^{2}}\right|_{\epsilon=0}\right. \\
& \left.+H_{y y}^{-}\left(B_{0}\right)\left(\left.\frac{\partial b_{\epsilon}}{\partial \epsilon}\right|_{\epsilon=0}\right)^{2}\right] .
\end{aligned}
$$

As the same, by $(2.3),(3.1)$ and the condition that $M_{1}(h) \equiv 0$, we can have

$$
M_{2}(h)=\left.\sum_{i=1}^{4} \frac{\partial^{2} l_{i}}{\partial \epsilon^{2}}\right|_{\epsilon=0} .
$$

Substituting (3.6), (3.7), (3.14), (3.15), (3.16) and (3.17) into (3.18), after a simple simplification, we obtain

$$
\begin{aligned}
M_{2}(h) & =\frac{H_{y}^{+}\left(A_{0}\right)}{H_{y}^{-}\left(A_{0}\right)}\left[\left.\frac{\partial^{2} l_{2}}{\partial \epsilon^{2}}\right|_{\epsilon=0}+\frac{H_{y y}^{-}\left(B_{0}\right)}{H_{y}^{-2}\left(B_{0}\right)}\left(\left.\frac{\partial l_{2}}{\partial \epsilon}\right|_{\epsilon=0}\right)^{2}\right] \\
& +\frac{H_{y}^{+}\left(A_{0}\right)}{H_{y}^{-}\left(A_{0}\right)} \frac{H_{y}^{-}\left(B_{0}\right)}{H_{y}^{+}\left(B_{0}\right)}\left[\left.\frac{\partial^{2} l_{4}}{\partial \epsilon^{2}}\right|_{\epsilon=0}-\frac{H_{y y}^{+}\left(B_{0}\right)}{H_{y}^{+2}\left(B_{0}\right)}\left(\left.\frac{\partial l_{4}}{\partial \epsilon}\right|_{\epsilon=0}\right)^{2}\right],
\end{aligned}
$$


where

$$
\begin{aligned}
&\left.\frac{\partial l_{4}}{\partial \epsilon}\right|_{\epsilon=0}=-\int_{\widehat{A_{0} B_{0}}} \omega^{+}, \\
&\left.\frac{\partial l_{2}}{\partial \epsilon}\right|_{\epsilon=0}=-\int_{\widehat{B_{0} A_{0}}} \omega^{-}, \\
&\left.\frac{\partial^{2} l_{4}}{\partial \epsilon^{2}}\right|_{\epsilon=0}=-\left.2 R_{y}^{+}\left(B_{0}\right) \frac{\partial b_{\epsilon}}{\partial \epsilon}\right|_{\epsilon=0}+2 \int_{\widehat{A_{0} B_{0}}} \psi^{+} \omega^{+}, \\
&\left.\frac{\partial^{2} l_{2}}{\partial \epsilon^{2}}\right|_{\epsilon=0}=\left.2 R_{y}^{-}\left(B_{0}\right) \frac{\partial b_{\epsilon}}{\partial \epsilon}\right|_{\epsilon=0}+2 \int_{\widehat{B_{0} A_{0}}} \psi^{-} \omega^{-} .
\end{aligned}
$$

Here it is easy to know that $\left.\frac{\partial b_{\epsilon}}{\partial \epsilon}\right|_{\epsilon=0}=-\frac{1}{H_{y}^{+}\left(B_{0}\right)} \int_{\widehat{A_{0} B_{0}}} \omega^{+}$and $R_{y}^{ \pm}=P^{ \pm}(x, y)-\psi^{ \pm}(x, y) H_{y}^{ \pm}(x, y)$. After a routine computation with (3.19), we can get (2.4). We have thus proved the theorem.

The proof of Corollary 2.1 will be omitted because it is analogous to that in Theorem 2.1.

\section{Application to piecewise quadratic systems}

It is already known that for piecewise quadratic perturbation of a linear center, the maximum number of the zeros of the first order Melnikov function $M_{1}(h)$ is equal to two[Liu \& Han, 2010]. We can prove the next proposition.

Proposition 4.1. We consider a piecewise polynomial system of the following form

$$
\left\{\begin{array}{l}
\dot{x}=y+\epsilon P^{+}(x, y), \\
\dot{y}=-x+\epsilon Q^{+}(x, y),
\end{array} \quad x>0, \quad\left\{\begin{array}{l}
\dot{x}=y+\epsilon P^{-}(x, y), \\
\dot{y}=-x+\epsilon Q^{-}(x, y),
\end{array} \quad x \leq 0,\right.\right.
$$

where the two perturbative terms are piecewise quadratic vector fields:

$$
\begin{gathered}
P^{+}(x, y)=a_{00}+a_{01} y+a_{10} x+a_{11} x y+a_{02} y^{2}+a_{20} x^{2} \\
Q^{+}(x, y)=b_{00}+b_{01} y+b_{10} x+b_{11} x y+b_{02} y^{2}+b_{20} x^{2}, \\
P^{-}(x, y)=c_{00}+c_{01} y+c_{10} x+c_{11} x y+c_{02} y^{2}+c_{20} x^{2}, \\
Q^{-}(x, y)=d_{00}+d_{01} y+d_{10} x+d_{11} x y+d_{02} y^{2}+d_{20} x^{2}
\end{gathered}
$$

For the perturbed piecewise Hamiltonian system (4.1) with $M_{1}(h) \equiv 0$, the maximum number of the zeros of the second order Melnikov function $M_{2}(h)$ is equal to three. We give here an example with such a maximum number of zeros.

Proof. The Hamiltonian functions of the unperturbed system (4.1) are $H^{+}(x, y)=H^{-}(x, y)=\frac{1}{2}\left(x^{2}+\right.$ $\left.y^{2}\right)=h$. It is obvious that $A_{0}(h)=(0, \sqrt{2 h})$ and $B_{0}(h)=(0,-\sqrt{2 h})$.

By the polar coordinate transformation of $x=\sqrt{2 h} \cos \theta, y=\sqrt{2 h} \sin \theta$, and (3.11), we obtain

$$
\begin{aligned}
M_{1}(h) & =-\left(\int_{\widehat{A_{0} B_{0}}} \omega^{+}+\int_{\widehat{B_{0} A_{0}}} \omega^{-}\right) \\
& =-\sum_{i+j=0}^{2}(\sqrt{2 h})^{i+j+1} \int_{\frac{\pi}{2}}^{-\frac{\pi}{2}}\left(a_{i j} \cos ^{i+1} \theta \sin ^{j} \theta+b_{i j} \cos ^{i} \theta \sin ^{j+1} \theta\right) d \theta \\
& -\sum_{i+j=0}^{2}(\sqrt{2 h})^{i+j+1} \int_{-\frac{\pi}{2}}^{-\frac{3 \pi}{2}}\left(c_{i j} \cos ^{i+1} \theta \sin ^{j} \theta+d_{i j} \cos ^{i} \theta \sin ^{j+1} \theta\right) d \theta \\
& =\sqrt{h}\left(A_{0}+A_{1} \sqrt{h}+A_{2}(\sqrt{h})^{2}\right),
\end{aligned}
$$


where

$$
\begin{aligned}
& A_{0}=2 \sqrt{2}\left(a_{00}-c_{00}\right) \\
& A_{1}=\pi\left(a_{10}+b_{01}+c_{10}+d_{01}\right) \\
& A_{2}=\frac{4 \sqrt{2}}{3}\left(2 a_{20}-2 c_{20}+a_{02}-c_{02}+b_{11}-d_{11}\right) .
\end{aligned}
$$

So $M_{1}(h)$ has at most two zeros. And with proper parameters, $M_{1}(h)$ can have exactly two zeros.

If $M_{1}(h) \equiv 0$, we have

$$
\begin{aligned}
& a_{00}=c_{00}, \\
& a_{10}+b_{01}+c_{10}+d_{01}=0, \\
& 2\left(a_{20}-c_{20}\right)+\left(a_{02}-c_{02}\right)+\left(b_{11}-d_{11}\right)=0 .
\end{aligned}
$$

Next we consider the second order Melnikov function, by Theorem 2.1, we simplify the formula as follows,

$$
\begin{aligned}
M_{2}(h) & =2\left(\int_{\widehat{A_{0} B_{0}}} \psi^{+} \omega^{+}+\int_{\widehat{B_{0} A_{0}}} \psi^{-} \omega^{-}+\left(P^{+}\left(B_{0}\right)-\psi^{+} H_{y}^{+}\left(B_{0}\right)\right) \frac{1}{H_{y}^{+}\left(B_{0}\right)} \int_{\widehat{A_{0} B_{0}}} \omega^{+}\right. \\
& \left.-\left(P^{-}\left(B_{0}\right)-\psi^{-} H_{y}^{-}\left(B_{0}\right)\right) \frac{1}{H_{y}^{+}\left(B_{0}\right)} \int_{\widehat{A_{0} B_{0}}} \omega^{+}\right) .
\end{aligned}
$$

According to the definition of $\psi^{ \pm}(x, y)$, we have

$$
\begin{aligned}
\psi^{+}(h, \theta) & =\int_{0}^{T^{+}(x, y)}\left(P_{x}^{+}+Q_{y}^{+}\right) d t \\
& =\int_{\frac{\pi}{2}}^{\theta} \sqrt{2 h}\left(2 a_{20} \cos \alpha+2 b_{02} \sin \alpha+a_{11} \sin \alpha+b_{11} \cos \alpha\right)+\left(a_{10}+b_{01}\right) d \alpha \\
& =\sqrt{2 h}\left(-a_{11} \cos \theta-2 b_{02} \cos \theta+2 a_{20} \sin \theta+b_{11} \sin \theta-2 a_{20}-b_{11}\right) \\
& -\frac{\pi}{2}\left(a_{10}+b_{01}\right)+\left(a_{10}+b_{01}\right) \theta .
\end{aligned}
$$

As the same calculation, we obtain

$$
\begin{aligned}
\psi^{-}(h, \theta) & =\int_{0}^{T^{-}(x, y)}\left(P_{x}^{-}+Q_{y}^{-}\right) d t \\
& =\int_{-\frac{\pi}{2}}^{\theta} \sqrt{2 h}\left(2 c_{20} \cos \alpha+2 d_{02} \sin \alpha+c_{11} \sin \alpha+d_{11} \cos \alpha\right)+\left(c_{10}+d_{01}\right) d \alpha \\
& =\sqrt{2 h}\left(-c_{11} \cos \theta-2 d_{02} \cos \theta+2 c_{20} \sin \theta+d_{11} \sin \theta+2 c_{20}+d_{11}\right) \\
& +\frac{\pi}{2}\left(c_{10}+d_{01}\right)+\left(c_{10}+d_{01}\right) \theta .
\end{aligned}
$$

Substituting $B_{0}(h)=(0,-\sqrt{2 h})$ into (4.4) and (4.5) gives to

$$
\psi^{+}\left(B_{0}\right)=-2 \sqrt{2 h}\left(2 a_{20}+b_{11}\right)-\pi\left(a_{10}+b_{01}\right), \quad \psi^{-}\left(B_{0}\right)=0 .
$$

Similarly, substituting $B_{0}(h)=(0,-\sqrt{2 h})$ into $P^{+}(x, y), P^{-}(x, y)$ and $H_{y}^{+}(x, y)$, we have

$$
P^{+}\left(B_{0}\right)=2 a_{02} h-a_{01} \sqrt{2 h}+a_{00}, \quad P^{-}\left(B_{0}\right)=2 c_{02} h-c_{01} \sqrt{2 h}+c_{00}, \quad H_{y}^{+}\left(B_{0}\right)=-\sqrt{2 h} .
$$

A routine computation with (4.3) gives rise to

$$
\begin{aligned}
M_{2}(h) & =2\left(\int_{\widehat{A_{0} B_{0}}} \psi^{+} \omega^{+}+\int_{\widehat{B_{0} A_{0}}} \psi^{-} \omega^{-}+\left(\frac{P^{+}\left(B_{0}\right)}{H_{y}^{+}\left(B_{0}\right)}-\psi^{+}\left(B_{0}\right)-\frac{P^{-}\left(B_{0}\right)}{H_{y}^{+}\left(B_{0}\right)}\right) \int_{\widehat{A_{0} B_{0}}} \omega^{+}\right) \\
& =\sqrt{h}\left(B_{0}+B_{1} \sqrt{h}+B_{2}(\sqrt{h})^{2}+B_{3}(\sqrt{h})^{3}\right),
\end{aligned}
$$


where

$$
\begin{aligned}
B_{0} & =4 \sqrt{2}\left(b_{00} c_{10}-a_{01} c_{00}+b_{00} d_{01}+c_{00} c_{01}+c_{10} d_{00}+d_{00} d_{01}\right) \\
B_{1} & =16\left(2 c_{00} c_{20}-b_{11} c_{00}+c_{00} d_{11}-2 a_{20} c_{00}\right)+\frac{\pi}{2}\left(4 c_{00} d_{02}-4 c_{20} d_{00}-3 c_{01} c_{10}\right. \\
& -4 a_{20} b_{00}+3 a_{01} c_{10}+3 a_{01} d_{01}-d_{10} d_{01}-d_{10} c_{10}+b_{10} c_{10}+b_{10} d_{01}-2 b_{00} b_{11} \\
& \left.-2 d_{00} d_{11}+2 c_{00} a_{11}+2 c_{00} c_{11}+4 c_{00} b_{02}-3 c_{01} d_{01}\right), \\
B_{2}= & -4 \sqrt{2} \pi\left(2 c_{10} c_{20}+2 c_{20} d_{01}+d_{11} c_{10}+d_{11} d_{01}-b_{11} d_{01}-2 a_{20} d_{01}-b_{11} c_{10}\right. \\
& \left.-2 a_{20} c_{10}\right)+\frac{4}{9} \sqrt{2}\left(d_{01} d_{02}+3 d_{10} d_{11}-5 c_{10} d_{02}-4 a_{11} c_{10}+12 c_{01} c_{20}-4 a_{11} d_{01}\right. \\
& +2 b_{20} d_{01}-6 a_{01} c_{20}-3 a_{01} c_{02}-5 b_{02} d_{01}-3 a_{01} d_{11}-3 b_{10} b_{11}+2 d_{20} d_{01}-3 a_{01} b_{11} \\
& -6 b_{01} b_{02}-4 c_{10} c_{11}-d_{01} c_{11}+2 b_{20} c_{10}+6 c_{01} d_{11}-6 a_{20} b_{10}+2 d_{20} c_{10}-3 a_{11} b_{01} \\
& \left.-6 a_{01} a_{20}-5 b_{02} c_{10}+3 c_{01} c_{02}+6 c_{20} d_{10}\right), \\
B_{3}= & \frac{32}{3}\left(4 c_{20} d_{11}-4 a_{20} c_{20}-2 a_{20} d_{11}-2 b_{11} c_{20}-b_{11} d_{11}+2 c_{02} c_{20}+4 c_{20}^{2}+c_{02} d_{11}\right. \\
& \left.+d_{11}^{2}-2 a_{20} c_{02}-b_{11} c_{02}\right)+\frac{\pi}{2}\left(2 b_{02} c_{02}+4 b_{02} c_{20}+2 b_{02} d_{11}-4 b_{02} a_{20}+2 a_{11} c_{20}\right. \\
& +a_{11} d_{11}-d_{20} d_{11}-a_{11} b_{11}+a_{11} c_{02}-a_{11} a_{20}+c_{02} c_{11}+c_{11} c_{20}-d_{02} d_{11}-b_{20} b_{11} \\
& \left.-2 b_{20} a_{20}+2 c_{02} d_{02}-3 b_{02} b_{11}-2 d_{20} c_{20}\right) .
\end{aligned}
$$

It is easy to see that $M_{2}(h)$ has at most three zeros, as we want to prove.

Consider system (4.1) with the following terms

$$
\begin{gathered}
P^{+}(x, y)=10+10 y-100 x+10^{3} x y+y^{2}+100 x^{2}, \\
Q^{+}(x, y)=-1+100 y+10 x+100 x y+y^{2}-10 x^{2}, \\
P^{-}(x, y)=10+y-x+x y+y^{2}+100 x^{2}, \\
Q^{-}(x, y)=1+y-x+100 x y+10 y^{2}-100 x^{2} .
\end{gathered}
$$

By directing calculation, $M_{1}(h) \equiv 0$ and

$$
M_{2}(h)=2 \sqrt{h}\left(-180 \sqrt{2}+10230 \pi \sqrt{h}-145184 \sqrt{2}(\sqrt{h})^{2}+\frac{133023 \pi}{2}(\sqrt{h})^{3}\right) .
$$

The zeros of $M_{2}(h)$ are equivalent to the zeros of $m_{2}(\sqrt{h})$, where

$$
m_{2}(\sqrt{h})=-180 \sqrt{2}+10230 \pi \sqrt{h}-145184 \sqrt{2}(\sqrt{h})^{2}+\frac{133023 \pi}{2}(\sqrt{h})^{3} .
$$

It is obvious to see that $m_{2}(\sqrt{h})$ has at most three zeros, and with the help of Maple software, it is easy to calculate that the three zeros are

$$
\sqrt{h_{1}} \approx 0.00836376959, \quad \sqrt{h_{2}} \approx 0.1844154805, \quad \sqrt{h_{3}} \approx 0.7898442362,
$$

see Fig. 3 for the graph of $m_{2}(\sqrt{h})$ with the condition $M_{1}(h) \equiv 0$. 


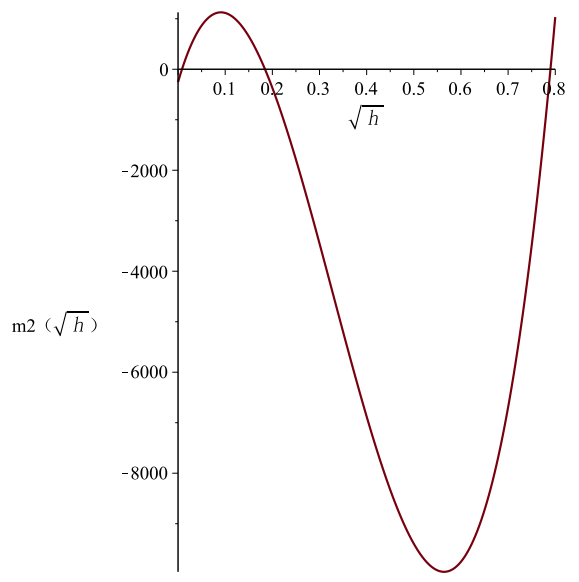

Fig. 3. The graph of $m_{2}(\sqrt{h})$ in $(4.7)$.

This completes the proof.

\section{Conclusion and Remarks}

Consider a system as follows,

$$
\left\{\begin{array}{l}
\dot{x}=y+\sum_{i=1}^{N} \epsilon^{i} P_{i}^{+}(x, y), \\
\dot{y}=-x+\sum_{i=1}^{N} \epsilon^{i} Q_{i}^{+}(x, y),
\end{array} \quad x>0, \quad\left\{\begin{array}{l}
\dot{x}=y+\sum_{i=1}^{N} \epsilon^{i} P_{i}^{-}(x, y), \\
\dot{y}=-x+\sum_{i=1}^{N} \epsilon^{i} Q_{i}^{-}(x, y),
\end{array} \quad x \leq 0,\right.\right.
$$

If $P_{i}^{+}(x, y)=P_{i}^{-}(x, y)$ and $Q_{i}^{+}(x, y)=Q_{i}^{-}(x, y)$, that is, the system is continuous. In [Iliev, 1999], for continuous quadratic perturbation of a linear center, it is shown that the maximum number of limit cycles is $0,1,1,2,2,3$, when $N=1,2,3,4,5,6$. Bautin in [Bautin, 1954] proved that, for continuous quadratic systems, it has at most three limit cycles near a focus or a center. Clearly, it can be found up to sixth order in $\epsilon$.

Furthermore, in [Buzzi et al., 2013], the authors consider systems for piecewise linear perturbations of a linear center, that is,

$$
P_{i}^{ \pm}(x, y)=a_{0 i}^{ \pm}+a_{1 i}^{ \pm} x+a_{2 i}^{ \pm} y, \quad Q_{i}^{ \pm}(x, y)=b_{0 i}^{ \pm}+b_{1 i}^{ \pm} x+b_{2 i}^{ \pm} y .
$$

They proved that the maximum number of limit cycles is $1,1,2,3,3,3,3$ when $N=1,2,3,4,5,6,7$, is the order of the Melnikov functions.

From Proposition 4.1 we can find three zeros of the second Melnikov function $M_{2}(h)$ up to the first order in $\epsilon$. In particular, consider system (5.8) with piecewise quadratic perturbation of a linear center up to second order in $\epsilon$, namely, $N=2$. According to Corollary 2.1, we can find that the maximum number of zeros (if $M_{1}(h) \equiv 0$ ) of the second Melnikov function $M_{2}(h)$ is 3 . Because the calculation is similar with Proposition 4.1, here we don't give the calculation process.

Consider a system as follows,

$$
\left\{\begin{array}{l}
\dot{x}=y(1+x)+\epsilon P(x, y) \\
\dot{y}=-x(1+x)+\epsilon Q(x, y)
\end{array}\right.
$$

where $P(x, y)=\sum_{i+j=0}^{n} a_{i j} x^{i} y^{j}$ and $Q(x, y)=\sum_{i+j=0}^{n} b_{i j} x^{i} y^{j}$. The unperturbed system of $(5.9)$ is formed by the linear center and a straight line of singular points. In [Llibre et al., 2001], the authors obtained that system (5.9) with $n=2$ and $a_{00}=b_{00}=0$, has 2 limit cycles by using averaging theory of first order. Later, Llibre and $\mathrm{Yu}$ [Llibre \& Yu, 2007] mentioned that system (5.9) with $a_{00}=b_{00}=0$, has at most 
$2 n-1$ limit cycles by the averaging theory of second order. But the upper bound can not be reached for $n=1,2,3$. Specially, it has one limit cycle for $n=2$. In [Buică et al., 2007], Buică et al. proved that system (5.9) with $n=2$ has 2 and 3 limit cycles by Melnikov functions of the second and third order, respectively. In fact, when we only consider a piecewise quadratic perturbation of a linear center, just like system (4.1), we can find 3 limit cycles of (4.1) by the second order Melnikov function.

To the best of our knowledge, there are also many articles studying piecewise perturbations of quadratic isochronous systems. In [Llibre \& Mereu, 2014], the authors obtained 5 limit cycles using the averaging theory of first order. Recently, the authors in [da Cruz et al., 2019] proposed a piecewise perturbation of quadratic differential system separated by a straight line, they obtained that the lower bound is 16 by the averaging theory of first and second order and they proved the existence of 16 crossing limit cycles.

In summary, we derive a formula for the second order Melnikov functions of piecewise Hamiltonian systems. An example shows that piecewise quadratic perturbation can have more limit cycles by the second order Melnikov functions than the smooth ones.

\section{Acknowledgments}

The corresponding author was supported by a grant of SJTU School of Mathematical Sciences. The first and third authors are supported by the state key program of NNSF of China grant number 11431008 and 11771282, NSF of Shanghai grant number 15ZR1423700.

\section{References}

Andronov, A. A., Vitt, A. A. \& Khaikin, S. E. [1966] Theory of oscillators, Translated from the Russian by F. Immirzi; translation edited and abridged by W. Fishwick (Pergamon Press, Oxford-New YorkToronto.).

Bautin, N. N. [1954] "On the number of limit cycles which appear with the variation of coefficients from an equilibrium position of focus or center type," American Math. Soc. Translation 100, 19 pp.(Russian original: Mat. Sb. 30, (1952), 181-196)

Buică, A., Gasull, A. \& Yang, J. [2007] "The third order Melnikov function of a quadratic center under quadratic perturbations," J. Math. Anal. Appl. 331, 443-454.

Buzzi, C., Pessoa, C. \& Torregrosa, J. [2013] "Piecewise linear perturbations of a linear center," Discrete Contin. Dyn. Syst. 33, 3915-3936.

Cardin, P. T. \& Torregrosa, J. [2016] "Limit cycles in planar piecewise linear differential systems with nonregular separation line," Phys. D 337, 67-82.

da Cruz, L. P. C., Novaes, D. D. \& Torregrosa, J. [2019] "New lower bound for the Hilbert number in piecewise quadratic differential systems," J. Differential Equations 266, 4170-4203.

Di Bernardo, M., Budd, C. J., Champneys, A. R. \& Kowalczyk, P. [2008] Piecewise-smooth dynamical systems. Theory and applications, Applied Mathematical Sciences, Vol. 163 (Springer-Verlag London, Ltd., London).

Françoise, J. P. [1996] "Successive derivatives of a first return map, application to the study of quadratic vector fields," Ergodic Theory Dynam. Systems 16, 87-96.

Freire, E., Ponce, E., Rodrigo, F. \& Torres, F. [1998] "Bifurcation sets of continuous piecewise linear systems with two zones," Internat. J. Bifur. Chaos Appl. Sci. Engrg. 8, 2073-2097.

Han, M. \& Zhang, W. [2010] "On Hopf bifurcation in non-smooth planar systems," J. Differential Equations 248, 2399-2416.

Huan, S.-M. \& Yang, X.-S. [2013] "Existence of limit cycles in general planar piecewise linear systems of saddle-saddle dynamics," Nonlinear Anal. 92, 82-95.

Iliev, I. D. [1999] "The number of limit cycles due to polynomial perturbations of the harmonic oscillator," Math. Proc. Cambridge Philos. Soc. 127, 317-322.

Liu, X. \& Han, M. [2010] "Bifurcation of limit cycles by perturbing piecewise Hamiltonian systems," Internat. J. Bifur. Chaos Appl. Sci. Engrg. 20, 1379-1390. 
Llibre, J. \& Mereu, A. C. [2014] "Limit cycles for discontinuous quadratic differential systems with two zones," J. Math. Anal. Appl. 413, 763-775.

Llibre, J., Mereu, A. C. \& Novaes, D. D. [2015a] "Averaging theory for discontinuous piecewise differential systems," J. Differential Equations 258, 4007 - 4032.

Llibre, J., Novaes, D. D. \& Teixeira, M. A. [2015b] "On the birth of limit cycles for non-smooth dynamical systems," Bull. Sci. Math. 139, 229-244.

Llibre, J., Pérez del Río, J. S. \& Rodríguez, J. A. [2001] "Averaging analysis of a perturbated quadratic center," Nonlinear Anal. 46, 45-51.

Llibre, J. \& Ponce, E. [2012] "Three nested limit cycles in discontinuous piecewise linear differential systems with two zones," Dyn. Contin. Discrete Impuls. Syst. Ser. B Appl. Algorithms 19, 325-335.

Llibre, J. \& Yu, J. [2007] "On the upper bound of the number of limit cycles obtained by the second order averaging method," Dyn. Contin. Discrete Impuls. Syst. Ser. B Appl. Algorithms 14, 841-873.

Lum, R. \& Chua, L. O. [1991] "Global properties of continuous piecewise linear vector fields. part i: Simplest case in $\mathbb{R}^{2}, "$ Internat. J. Circuit Theory Appl. 19, 251-307.

Makarenkov, O. \& Lamb, J. S. W. [2012] "Dynamics and bifurcations of nonsmooth systems: a survey," Phys. D 241, 1826-1844. 